\title{
Tuberculose: os futuros profissionais de saúde são preparados para o atendimento adequado do paciente?
}

\author{
Tuberculosis: are the future health care professionals \\ prepared for adequate patient care?
}

\section{Gabriela dos Santos Paschoal', Gabriela Santos de Souza ${ }^{2}$, Mariele Pereira ${ }^{3}$, Luisa Patrícia Fogarolli de Carvalho ${ }^{4}$}

\author{
'Autora para correspondência. Universidade Federal de Alfenas, Alfenas, Minas Gerais, Brasil. \\ ORCID: 0000-0002-6829-8101. gabrielapaschoal-@hotmail.com \\ ${ }^{2}$ Universidade Federal de Alfenas, Alfenas, Minas Gerais, Brasil. ORCID: 0000-0002-4145-7248. gabrielasouza195@hotmail.com \\ 3Universidade Federal de Alfenas, Alfenas, Minas Gerais, Brasil. ORCID: 0000-0001-8986-1138. mariellepereira-14@hotmail.com \\ ${ }^{4}$ Universidade Federal de Alfenas, Alfenas, Minas Gerais, Brasil. ORCID: 0000-0002-3770-8833. luisa.fogarolli@gmail.com
}

RESUMO I INTRODUÇÃO: Segundo a Organização Mundial da Saúde (OMS), um terço da população mundial está infectada pelo Mycobacterium tuberculosis'. A literatura mostra que a equipe multiprofissional responsável pelo manejo da tuberculose não está apta a lidar frente à reemergência da doença, além de atuarem de forma não integral e humanizada. OBJETIVO: Verificar se os universitários que serão inseridos na atenção primária estão aptos a identificar e manejar pacientes com tuberculose. Métodos e materiais: Foram aplicados questionários semiestruturados em alunos dos últimos períodos dos cursos da área da saúde. Os dados foram analisados através de estatística descritiva. RESULTADOS: A maioria dos entrevistados disseram já ter tido contato com o tema tuberculose durante a graduação. Em relação às manifestações, $65(38,24 \%)$ consideraram como principais sintomas da tuberculose pulmonar tosse, emagrecimento, febre, perda de apetite, sudorese noturna e fraqueza. Em contrapartida, $97(57,06 \%)$ consideraram a presença do escarro hemoptoico como manifestação principal. A minoria dos entrevistados soube definir corretamente um sintomático respiratório. CONCLUSÃO: Através da análise dos resultados é possível notar que a universidade é a principal fonte de conhecimento dos universitários sobre tuberculose. Identificou-se lacunas a serem preenchidas, como em relação às manifestações da doença, à dificuldade em identificar um sintomático respiratório, o desconhecimento da mudança do calendário vacinal e da obrigatoriedade da realização do teste rápido de HIV. Além disso, percebe-se que há dificuldade no manejo adequado dos pacientes. Diante dos resultados, é evidenciada a necessidade de aprimoramento do ensino em relação à tuberculose.

PALAVRAS-CHAVE: Tuberculose. Conhecimento. Estudantes.
ABSTRACT I INTRODUCTION: According to the World Health Organization (WHO), one third of the world's population is infected with Mycobacterium tuberculosis'. The literature shows that the multiprofessional team responsible for managing tuberculosis is not able to dealing with re-emergence of the disease, in addition acting in non-integral and humanized way. OBJECTIVE: To check if university students that will be inserted in primary care are able to identify and manage patients with tuberculosis. METHODS AND MATERIALS: Semi-structured questionnaires were applied to students of the last periods of health courses. Data were analyzed through descriptive statistics. RESULTS: Most of the interviewees said they have already had contact with the topic tuberculosis during graduation. Regarding the manifestations, $65(38.24 \%)$ considered cough, weight loss, fever, loss of appetite, nocturnal sweating and weakness as the main symptoms of pulmonary tuberculosis. In contrast, $97(57.06 \%)$ considered the presence of hemoptoic sputum as the main manifestation. The minority of the interviewees knew how to correctly define a respiratory symptomatic subject. CONCLUSION: Through the analysis of the results it is possible to notice that the university is the main source of knowledge of the university students about tuberculosis. One had identified gaps to be filled, as in relation to the manifestations of the disease, the difficulty in identifying a respiratory symptomatic subject, the lack of knowledge of the change in the vaccination schedule and mandatory rapid HIV testing. In addition, it is noticed that there is difficulty in the adequate management of the patients. In view of the results, there is a need to improve teaching in relation to tuberculosis.

KEYWORDS: Tuberculosis. Knowledge. Students. 


\section{Introdução}

Segundo a Organização Mundial de Saúde, um terço da população mundial está infectada pelo Mycobacterium tuberculosis, sendo contabilizados, em 2013, 9 milhões de casos novos e 1 milhão de óbitos'. Cerca de $80 \%$ desses casos estão concentrados em 22 países, dentre os quais o Brasil que ocupa a $16^{a}$ posição em número de casos absolutos, sendo responsável por $31 \%$ dos casos de tuberculose na América Latina ${ }^{2}$.

No Brasil, foram diagnosticados aproximadamente 70.000 novos casos de tuberculose em 2014, com uma redução da taxa de incidência, passando de 41,5/100 mil habitantes (hab.) em 2005 para 33,5 por 100 mil hab. em $2014^{3}$.

A tuberculose apresenta um longo período de latência, acometendo principalmente os pulmões, sendo os pacientes que apresentam a forma pulmonar bacilífera potenciais transmissores ${ }^{4,5}$. São considerados sintomáticos respiratórios os que apresentam tosse por três semanas ou mais, os quais, muitas vezes, são negligenciados pelos profissionais de saúde ${ }^{6,7}$.

As manifestações clínicas da tuberculose podem se apresentar de forma aguda e grave (menos comum) ou de forma mais insidiosa e lenta (mais comum). $\mathrm{Na}$ forma insidiosa os principais sintomas são febre baixa, sudorese noturna, inapetência e exame físico inexpressivo ${ }^{8}$. O diagnóstico da tuberculose é feito principalmente pela baciloscopia do escarro, a qual é indicada para todos os sintomáticos respiratórios e também para monitoramento durante o tratamento, sendo obrigatório ao término dos $2^{\circ}, 4^{\circ}$ e $6^{\circ}$ mês de tratamento, pela identificação dos bacilos através da cultura e método moleculares ${ }^{5,9}$.

Conforme os dados encontrados no trabalho de Oliveira et $\mathrm{al}^{7}$, apesar do acolhimento dos casos suspeitos ter sido realizado na atenção primária, o diagnóstico da tuberculose foi realizado na atenção secundária e terciária em $73 \%$ dos pacientes, com apenas $16 \%$ ocorrendo na atenção primária.

A coinfecção pelos vírus da imunodeficiência humana (HIV) e Mycobacterium tuberculosis é responsável pela dificuldade na redução da incidência de ambas as infecções. Essa relação teve grande impacto nos programas de controle da tuberculose, pois 0 HIV tem contribuindo tanto para o aumento do número de casos de tuberculose quanto para a elevação das taxas de mortalidade entre os pacientes co-infectados ${ }^{5,4}$.

A equipe multiprofissional, formada minimamente por médico, enfermeiro, agente comunitário de saúde e técnico de enfermagem, desempenha importante papel no manejo da tuberculose, tendo como funções busca ativa e identificação dos sintomáticos respiratórios, orientação com relação ao tratamento e enfrentamento da doença e estratégias para a realização de diagnóstico precoce. Isso é dificultado pela "inabilidade dos profissionais de saúde" em identificar a doença, seus sinais e sintomas, além da atuação de forma não integral e humanizada, pela sobrecarga dos serviços de saúde e a falta de investimentos em uma política de saúde pública organizada se tornam um empecilho para essa investigação ${ }^{10-12}$.

A Organização Mundial de Saúde (OMS) demonstra preocupação em relação à qualificação das equipes de saúde no que diz respeito as ações de controle da doença, principalmente no âmbito da Atenção Primária à Saúde ${ }^{13}$. Diante disso, surge a necessidade de os gestores oferecerem educação contínua aos profissionais que atuam diretamente no Programa de Controle da Tuberculose ${ }^{10}$.

Com isso, este trabalho objetivou analisar o conhecimento dos universitários da área de saúde da Universidade Federal de Alfenas-MG (UNIFAL-MG) acerca da tuberculose para avaliar se estão aptos para o manejo da tuberculose, visto que o número de trabalhos produzidos abordando esse tema são reduzidos, englobando apenas alguns profissionais da equipe básica de saúde. A importância deste trabalho se dá pelo fato de que a partir dos dados obtidos através desta pesquisa haverá mais informações disponíveis que permitam a implementação de intervenções visando aumentar/sedimentar o conhecimento sobre o tema levando posteriormente a um melhor atendimento para a comunidade. 


\section{Métodos e materiais}

A pesquisa, realizada entre os meses de abril e maio de 2017, consistiu em um estudo populacional quantitativo descritivo, através da utilização de um questionário semiestruturado abrangendo a transmissão, sintomatologia, diagnóstico, prevenção e tratamento da tuberculose, sendo direcionado aos alunos dos anos finais dos cursos da saúde. O questionário foi validado por uma infectologista, um pneumologista e uma médica de família, sendo acatadas as sugestões de aprimoramento. Foram excluídos desse estudo aqueles que não tinham mais atividades no campus devido à dedicação exclusiva ao estágio, pela impossibilidade da aplicação do questionário presencial.
A amostra inicial foi composta por 218 estudantes dos últimos períodos dos cursos de Enfermagem, Farmácia, Fisioterapia, Medicina, Nutrição e Odontologia, contudo 170 estudantes participaram efetivamente da pesquisa (Tabela 1). Os dados foram analisados através de estatística descritiva, construção de tabelas e gráficos através da utilização do software Excel Office ${ }^{\circledR}$.

Projeto de Pesquisa encontra-se em consonância com a Resolução 466/2012 do Conselho Nacional de Saúde do Ministério da Saúde e foi aprovado pelo Comitê de Ética em Pesquisa da UNIFAL-MG, número do parecer de aprovação do Comitê de Ética e Pesquisa: 1.854.0144.

A pesquisa foi realizada apenas com os alunos que assinaram $\circ$ Termo de Consentimento Livre e Esclarecido (TCLE).

Tabela 1. Número de alunos por curso e período ao qual foi aplicado o questionário

\begin{tabular}{lcc}
\hline \multicolumn{1}{c}{ Curso } & Período a ser aplicado o questionário & Número de alunos \\
\hline \hline Enfermagem & $9^{\circ}$ período & 21 \\
Farmácia & $9^{\circ}$ período & 24 \\
Fisioterapia & $9^{\circ}$ período & 36 \\
Medicina & $7^{\circ}$ período & 27 \\
Nutrição & $7^{\circ}$ período & 28 \\
Odontologia & $9^{\circ}$ período & 34 \\
TOTAL & & 170 \\
\hline
\end{tabular}

Fonte: Os autores (2018).

\section{Resultados}

A idade dos entrevistados variou entre 20 e 38 anos (média de 23,49 anos). Destes, 150 (88,24\%) disseram já ter tido contato com o tema tuberculose, a maioria $128(75,29 \%)$ na graduação, principalmente através de disciplinas obrigatórias.

Quanto a forma de transmissão, 95 entrevistados $(55,88 \%)$ responderam que ela ocorre através de aerossóis contendo o bacilo e 32 (18,83\%) por compartilhamento de talheres e copos. Quando perguntado sobre quais pacientes podem transmitir a tuberculose, $87(51,18 \%)$ responderam que os potenciais transmissores são os que apresentam a forma pulmonar com eliminação do bacilo. Dentre os entrevistados, 132 (77,65\%) souberam responder que nem todas as pessoas que entram em contato com o agente etiológico desenvolvem a doença (Tabela 2). 


\begin{tabular}{lc}
\hline \multicolumn{1}{c}{ QUESTIONÁRIO/RESPOSTAS } & N $(\%)$ \\
\hline \hline Qual a forma de transmissão da Tuberculose? & $32(18,83)$ \\
a. Através do contato com talheres/copos/outros utensílios & $7(4,12)$ \\
b. Através do beijo/abraço/relações sexuais & $95(55,88)$ \\
c. Através de aerossóis que contenha o bacilo & $16(9,41)$ \\
d. Através da ingestão de alimentos/água contaminados com o bacilo & $5(2,94)$ \\
e. Não sei & $15(8,82)$ \\
Nulo & $15(8,81)$ \\
Quais os pacientes podem transmitir a Tuberculose? & $87(51,18)$ \\
a. Todos os pacientes portadores do bacilo & $11(6,47)$ \\
b. Pacientes com a forma pulmonar, que estejam eliminado o bacilo & $36(21,18)$ \\
c. Pacientes com a forma pulmonar, mesmo que não estejam eliminando o bacilo & já tenham \\
d. Pacientes que apresentaram a forma ativa da doença, mesmo que & $14(8,24)$ \\
iniciado o tratamento a mais de 15 dias & $7(4,12)$ \\
e. Não sei & \\
Nulo &
\end{tabular}

Fonte: Os autores (2018).

Em relação às manifestações clínicas, $65(38,24 \%)$ consideraram como principais sintomas da tuberculose pulmonar tosse, emagrecimento, febre, perda de apetite, sudorese noturna e fraqueza. Em contrapartida, 97 $(57,06 \%)$ consideraram presença do escarro hemoptoico como manifestação principal (Tabela 3). Sobre a definição de um sintomático respiratório, $64(37,65 \%)$ julgaram ser aquele paciente que apresenta tosse há três semanas ou mais.

Tabela 3. Resultados obtidos na realização da pesquisa $(N=170)$

\begin{tabular}{lc}
\hline \multicolumn{1}{c}{ QUESTIONÁRIO/RESPOSTAS } & N $(\%)$ \\
\hline \hline Quais são os principais sintomas da Tuberculose Pulmonar? & $97(57,06)$ \\
a. Tosse, emagrecimento, diarreia, febre, sudorese noturna e escarro com sangue & $0(0,00)$ \\
b. Tosse, ganho de peso, aumento do apetite, febre e dores no peito & $3(1,76)$ \\
c. Tosse, emagrecimento, queda de cabelo, fraqueza, diarreia e febre & $3(1,76)$ \\
d. Tosse, ganho de peso, febre, dispneia, dores nas costas e no peito & $65(38,24)$ \\
e. Tosse, emagrecimento, febre, perda de apetite, sudorese noturna e fraqueza & $2(1,18)$ \\
Nulo & \\
\hline
\end{tabular}

Fonte: Os autores (2018). 
Quanto ao diagnóstico da tuberculose pulmonar, $100(58,82 \%)$ apontaram ser necessário a existência de duas baciloscopias positivas de escarro e um exame de raio-X. Para a realização da baciloscopia de escarro, $92(54,12 \%)$ indicaram adequadamente as medidas de biossegurança. Em relação a realização do teste rápido para HIV nos pacientes diagnosticados com tuberculose, 76 $(44,70 \%)$ consideraram obrigatória a realização deste teste e $73(42,94 \%)$ não sabiam sobre a obrigatoriedade do mesmo.
No que se refere a prevenção, 27 (15,88\%) consideraram a alimentação e condições de habitação adequadas, vacinação com BCG e tratamento de infecção latente como medidas preventivas para a tuberculose. Encontramos que $67(39,42 \%)$ consideraram ser necessário evitar compartilhar utensílios com os doentes. Sobre a imunização com a vacina BCG, 25 (14,71\%) disseram que a proteção ocorre somente contra as formas graves de tuberculose, contudo, $62(36,47 \%)$ acreditam que a vacina protege somente por determinado período de tempo, sendo necessário reforço após dez anos da primeira dose (Tabela 4).

Tabela 4. Resultados obtidos na realização da pesquisa $(N=170)$

\begin{tabular}{|c|c|}
\hline QUESTIONÁRIO/RESPOSTAS & $\mathbf{N}(\%)$ \\
\hline \multicolumn{2}{|l|}{ Quais as formas de prevenção da Tuberculose? } \\
\hline $\begin{array}{l}\text { a. Alimentação e condições de habitação adequadas, vacinação com BCG e evitar } \\
\text { compartilhar utensílios com os doentes }\end{array}$ & $67(39,42)$ \\
\hline $\begin{array}{l}\text { b. Alimentação e condições de habitação adequadas, vacinação com BCG, } \\
\text { quimioprofilaxia e isolamento do doente em lugares frios }\end{array}$ & $5(2,94)$ \\
\hline \multicolumn{2}{|l|}{ vacinação com BCG } \\
\hline quimioprofilaxia e evitar compartilhar utensílios com os doentes & $49(28,82)$ \\
\hline $\begin{array}{l}\text { e. Alimentação e condições de habitação adequadas, vacinação com BCG e } \\
\text { quimioprofilaxia }\end{array}$ & $27(15,88)$ \\
\hline Nulo & $7(4,12)$ \\
\hline \multicolumn{2}{|l|}{ A vacina $B C G$ : } \\
\hline a. Protege contra todas as formas de tuberculose & $52(30,58)$ \\
\hline b. Protege somente pacientes imunodeprimidos & $2(1,18)$ \\
\hline c. Protege somente contra as formas graves de tuberculose & $25(14,71)$ \\
\hline d. Protege somente contra a forma pulmonar da tuberculose & $22(12,94)$ \\
\hline e. Protege somente por determinado período de tempo, sendo necessário reforço após & $62(36,47)$ \\
\hline \multicolumn{2}{|l|}{ dez anos da primeira dose } \\
\hline Nulo & $7(4,12)$ \\
\hline
\end{tabular}

Fonte: Os autores (2018). 
Sobre o tratamento supervisionado (DOTS), 66 $(38,82 \%)$ consideram que a supervisão pode ser feita apenas por profissionais da saúde, enquanto $22(12,94 \%)$ consideram que a comunidade pode contribuir com a equipe de saúde.

No que tange $\circ$ esquema terapêutico para a tuberculose pulmonar não complicada, 101 (59,41\%) consideraram adequado o uso de rifampicina, isoniazida, pirazinamida e etambutol por 6 meses.

Analisando ○ papel da vigilância epidemiológica na obtenção de dados para o controle e implantação de programas de prevenção da tuberculose, $133(78,24 \%)$ responderam que deve ser realizada investigação epidemiológica entre os contatos de todo caso novo de tuberculose, sendo uma doença de notificação compulsória e investigação obrigatória. Quando questionados sobre a tuberculose ser ou não uma doença curável, 146 (85,87\%) consideraram existir a cura para a tuberculose.

Dos 170 entrevistados, somente 165 responderam o item referente a considerar-se apto ou não apto ao atendimento da tuberculose na atenção primária. Destes, $84(50,91 \%)$ se consideraram preparados para lidar com a tuberculose e 81 (49,09\%) discordam estar aptos a lidar com a tuberculose.

\section{Discussão}

A cura do paciente com tuberculose resulta da associação medicamentosa adequada, considerando doses e tempo corretos, evitando a persistência bacteriana e o desenvolvimento de resistência aos fármacos 6 . Em nosso estudo, quando indagados sobre a possibilidade de cura da tuberculose a maioria dos pesquisados a consideraram como uma patologia curável, o que é compatível com os resultados obtidos por Sánchez, em que $78,3 \%$ dos participantes da pesquisa tiveram a mesma resposta ${ }^{14}$.

A transmissão ocorre por via aérea em praticamente todos os casos, a partir da inalação de partículas contendo bacilos expelidos e se inicia simultaneamente aos primeiros sintomas respiratórios, decaindo após o início de tratamento efetivo ${ }^{6}$. De acordo com Secretaria de Estado de Saúde de Minas
Gerais, as gotículas que se depositam em objetos como roupas, lençóis e utensílios domésticos dificilmente se dispersarão em aerossóis e assim não são fator importante na transmissão ${ }^{8}$.

Nota-se em nossos resultados que há ainda muitas dúvidas em relação à transmissão da tuberculose, - que condiz com o estudo de Mussi, em que mais de $90 \%$ dos participantes da pesquisa apontaram como forma de contágio da doença o uso compartilhado de utensílios, como copos e talheres. No mesmo trabalho $60 \%$ dos estudantes disseram ser a fala uma forma de transmissão ${ }^{15}$. Já no trabaIho de Teixeira, metade dos estudantes de medicina identificou como forma de transmissão fala, tosse e espirro, sendo sugerido que deve haver melhorias no conhecimento dos estudantes sobre a transmissão da tuberculose ${ }^{16}$.

Embora metade dos entrevistados soubesse definir quais pacientes são potenciais transmissores da doença, uma parcela de $36(21,18 \%)$ dos pesquisados apontaram que até mesmos os pacientes que tenham iniciado o tratamento a mais de 15 dias são potenciais transmissores, o que coincide com o que diz Mussi, em que 85,5\% dos entrevistados responderam que a contaminação pelo bacilo da tuberculose é possível ao falar com pessoas com tuberculose que estejam recebendo tratamento por mais de um mês ${ }^{15}$.

Segundo o Manual de Recomendações para o Controle da Tuberculose no Brasil, a maioria das pessoas que são expostas ao bacilo resiste ao adoecimento e adquirem imunidade parcial à doença ${ }^{6}$. No estudo de Sánchez, 40,6\% dos entrevistados, apontaram que "qualquer um" pode contrair tuberculose e apenas 10,3\% disseram que "pessoas com baixas defesas" são mais suscetíveis à doença ${ }^{14}$. Em nosso estudo, a maioria dos entrevistados acreditam que nem todo os indivíduos que entram em contato com o agente etiológico adoecem, demonstrando que conseguem reconhecer a tuberculose como uma doença com predileção para populações especiais.

Quanto às manifestações clínicas, a minoria da amostra $(38,24 \%)$ soube identifica-las corretamente, o que corresponde aos achados de Sobrinho, em que somente $16,7 \%$ dos profissionais entrevistados souberam indicar os sinais clássicos da doen- 
ça corretamente ${ }^{11}$. Basu identificou que $64,4 \%$ dos médicos estagiários mencionaram a tosse por duas semanas ou mais como principal sintoma da tuberculose, citando também a hemoptise ${ }^{17}$. No estudo de Al-Jabri $57 \%$ dos estudantes de medicina disseram que todo paciente com tuberculose apresenta tosse com escarro hemoptoico ${ }^{18}$. Isso evidencia que a dificuldade em identificar os pacientes com tuberculose está presente tanto na vida acadêmica, quanto na profissional.

O sintomático respiratório é definido como o indivíduo que apresenta tosse há mais de três semanas, contudo em populações especiais há redução para duas semanas ${ }^{6}$. Em nosso estudo, $37,65 \%$ dos entrevistados consideraram sintomático respiratório a presença de tosse há mais de três semanas, enquanto $28,82 \%$ consideram a presença de tosse há mais de duas semanas.

O diagnóstico definitivo da tuberculose é feito através da realização da baciloscopia direta de escarro e exame radiológico, solicitado a todo paciente com suspeita clínica da doença ${ }^{6}$. A coleta da amostra para a realização da baciloscopia do escarro deve ser realizada em ambiente aberto e ventilado, com uso da máscara N95 pelos profissionais ${ }^{8}$.

A maioria dos entrevistados apontaram ser necessária a presença de duas baciloscopias de escarro positivas e um exame de raio-X para comprovação diagnóstica. Para a realização da baciloscopia de escarro, $54,12 \%$ consideraram que a coleta deve ser realizada em ambiente aberto e ventilado, com uso de máscara N95 pelo profissional de saúde. Isso contrapõe os achados de Mussi, em que $100 \%$ dos estudantes não mencionaram a baciloscopia como primeiro teste para diagnóstico da tuberculose pulmonar ativa e $97,2 \%$ dos estudantes afirmaram o uso da máscara N95 ser uma forma de prevenção contra a tuberculose ativa ${ }^{15}$. Já no trabalho de Temesgen, apenas $34,2 \%$ identificaram $\circ$ uso de máscara pelos profissionais de saúde como medida preventiva, embora somente $21,1 \%$ afirmam fazer uso da mesma ${ }^{19}$.

A prevenção da doença acontece através da busca ativa de casos novos, tratamento adequado, controle de contactantes, ações educativas para a população e profissionais de saúde e da vacinação com BCG, a qual não evita o desenvolvimento do quadro patológico, mas sim evita o desenvolvimento de formas graves ${ }^{8,20}$. Em nossa pesquisa, apenas $15,88 \%$ dos entrevistados consideraram condições de alimentação e habitação, que inclui ambiente ventilado, vacinação com $B C G$ e quimioprofilaxia (tratamento de infecção latente) como medidas preventivas da tuberculose. Isso evidencia que é necessário maior enfoque nas medidas educativas em relação às formas de prevenção da tuberculose durante a graduação.

No que tange a proteção contra a tuberculose através da imunização com a vacina $B C G$, somente 25 $(14,71 \%)$ responderam adequadamente que a imunização ocorre apenas para as formas graves da doença. Nossos achados correspondem ao trabalho de Mussi, em que $95,7 \%$ dos participantes responderam incorretamente ao serem questionados sobre a eficácia da vacina da BGC contra a tuberculose pulmonar, e de Al-Jabri, em que $88 \%$ dos estudantes de medicina consideraram a vacina BCG como forma de proteção $100 \%$ efetiva em todos os casos de tuberculose ${ }^{15,18}$. Uma possível causa para esse considerável índice de erros é a mudança do calendário vacinal determinada pela Portaria $\mathrm{N}^{\circ} 1602$ de 17 de julho de 2006, em que deixou de ser conduta a aplicação da dose de reforço da vacina aos 10 anos de idade, visto que a média de idade da população estudada é de 23,49 anos, ou seja, a maioria recebeu a segunda dose da vacina ${ }^{21}$. Tal divergência mostra que é necessário maior enfoque nas atualizações a respeito do calendário vacinal na graduação.

A alta prevalência da coinfecção tuberculose e HIV, fez com que se tornasse obrigatório a utilização do teste rápido anti-HIV em pacientes diagnosticados com tuberculose ${ }^{6}$. Com a análise dos dados foi possível notar que menos da metade $(44,70 \%)$ dos entrevistados sabem da obrigatoriedade da realização do teste rápido para HIV em pacientes com suspeita de tuberculose, fato que gera preocupação, pois a ascensão da tuberculose está intrinsecamente ligada ao aumento do número de casos de $\mathrm{HIV}^{4}$.

O Tratamento Diretamente Observado (DOT) pode ser feito em Unidades de Saúde, na residência do doente e em outros locais a combinar, sendo a supervisão feita por profissionais do Programa de Saúde 
da Família, familiares e pessoas da comunidade ${ }^{7}$. Em nosso estudo apenas $12,94 \%$ dos participantes souberam definir no que consiste o DOT, isso corrobora com o estudo de Sobrinho, em que a maioria dos profissionais também não tinha conhecimento sobre o $D O T^{11}$. Esse cenário demonstra preocupação em relação à continuidade do tratamento, por este ser de longa duração enquanto que a melhora dos sintomas é percebida em poucas semanas, favorecendo $o$ abandono.

Um dos fatores que predispõe o abandono é a transmissão inadequada de informações sobre o tempo de tratamento pelos profissionais de saúde, o que condiz com o que diz Vieira, que identificou maior taxa de abandono em pacientes com tratamento auto-administrados do que naqueles com 0 DOT $^{22,23}$. Isso ressalta a importância da educação da comunidade e dos profissionais para maior compreensão do papel da população no manejo e controle da tuberculose.

Com relação ao esquema básico terapêutico para o tratamento da tuberculose, o Ministério da Saúde preconiza que $\circ$ mesmo seja feito em duas fases, sendo a primeira fase intensiva, com os medicamentos rifampicina, isoniazida, pirazinamida e etambutol, de duração de 2 meses e a segunda fase de manutenção, composta por rifampicina e isoniazida, com duração de 4 meses, totalizando 6 meses de tratamento ${ }^{6}$. A maioria dos pesquisados apontou a necessidade de tratamento durante 6 meses em casos de tuberculose pulmonar não complicada, diferindo do que diz Sánchez, no qual $41,7 \%$ dos entrevistados afirmaram que o tratamento deve durar aproximadamente 9 meses $^{14}$. Nos achados de Montagna, $75 \%$ dos entrevistados consideram necessário o uso de uma combinação de antibióticos por um longo período para a efetividade do tratamento ${ }^{24}$. Por outro lado Al-Jabri, 94\% dos estudantes de medicina julgam que duas semanas de tratamento com antibióticos são suficientes para se obter a cura ${ }^{18}$.

De acordo com a Portaria $\mathrm{n}^{\circ}$ 104, de 25 de janeiro de 2011 , a tuberculose é agravo de notificação compulsória, sendo que todos os casos novos, recidivas e abandono devem ser notificados por meio da ficha do Sistema de Agravos de Notificação - SINAN, devendo ser feita a investigação epide- miológica dos contactantes ${ }^{6}$. Em nossa pesquisa, a maioria dos entrevistados soube que essa conduta deve ser realizada.

\section{Conclusão}

Com a realização deste trabalho, foi possível identificar que os estudantes da área da saúde apresentam muitas lacunas a serem preenchidas no que diz respeito à tuberculose, identificadas principalmente na caracterização dos sintomas, formas de prevenção, imunização, realização do teste rápido de HIV e definição do DOT. Além disso, percebe-se que as atualizações a respeito da doença não estão sendo acompanhadas de forma efetiva no ensino superior, o que pode contribuir para as dificuldades encontradas pelos profissionais em sua prática clínica. Assim, destaca-se a necessidade do fortalecimento do ensino em relação a tuberculose e implementação de métodos de ensino que contribuam para a fixação do conhecimento.

\section{Contribuições dos autores}

Paschoal GS participou da concepção, delineamento, busca e análise estatística dos dados da pesquisa, interpretação dos resultados, redação do artigo científico e encaminhamento do artigo científico. Souza GS participou da concepção, delineamento, busca e análise estatística dos dados da pesquisa, interpretação dos resultados, redação do artigo científico e encaminhamento do artigo científico. Pereira M participou da concepção, delineamento, busca e análise estatística dos dados da pesquisa, interpretação dos resultados, redação do artigo científico e encaminhamento do artigo científico. Carvalho LPF participou da concepção, delineamento, interpretação dos resultados e redação do artigo científico.

\section{Conflitos de interesses}

Nenhum conflito financeiro, legal ou político envolvendo terceiros (governo, empresas e fundações privadas, etc.) foi declarado para nenhum aspecto do trabalho submetido (incluindo mas não limitandose a subvenções e financiamentos, conselho consultivo, desenho de estudo, preparação de manuscrito, análise estatística, etc).

\section{Referências}

1. World Health Organization. Global tuberculosis report 2014. Geneva; 2014. 
2. Angelotti LCZ, Alexandre PBD, Miranzi SSC, Scatena LM. Quality of report data and the follow-up of tuberculosis cases in Minas Gerais. REAS. 2013;2(2 NEsp):84-98.

3. Ministério da Saúde. Secretaria de Vigilância em Saúde. Boletim Epidemiológico [Internet]. 2015 [acesso em 2018 Ago 29]. Disponível em: http://portalarquivos.saude.gov.br/ images/pdf/2015/marco/25/Boletim-tuberculose-2015.pdf

4. Nogueira AF, Facchinetti V, Souza MVN, Vasconcelos TRA. Tuberculosis: a general approach of the main aspects. Rev Bras Farm. 201 2;93(1):3-9.

5. Ministério da Saúde. Secretaria de Vigilância em Saúde. Departamento de Vigilância Epidemiológica. Infectious and Parasitic Diseases: pocketguide. 8.ed. Brasília, DF: Ministério da Saúde; 2010.

6. Ministério da Saúde. Secretaria de Vigilância em Saúde. Departamento de Vigilância Epidemiológica. Guidelines for tuberculosis control in Brazil. Brasília, DF: Ministério da Saúde; 2011.

7. Oliveira MF, Arcêncio RA, Ruffino-Netto A, Scatena LM, Palha PF, Villa TCS. The front door of the Ribeirão Preto Health System for diagnosing tuberculosis. Rev Esc Enferm USP. 2011 ;45(4):898-904. doi: 10.1590/S0080$\underline{62342011000400015}$

8. Secretaria de Estado de Saúde de Minas Gerais. Atenção à Saúde do Adulto: Tuberculose. Belo Horizonte: Secretaria de Estado de Saúde de Minas Gerais; 2006.

9. Ferri AO, Aguiar B, Wilhelm CM, Schmidt D, Fussieger F, Picoli SU. Diagnóstico da tuberculose: uma revisão. Revista Liberato. 15(24);105-212.

10. Furlan MCR, Silva RLDT, Marcon SS. Factors associated with early and late diagnosis of tuberculosis: a descriptive study. Online Braz J Nurs. 2013;13(1):62-71. doi: $10.5935 / 1676-4285.20144364$

11. Sobrinho RASS, Souza AL, Silva LMC, Wysocki AD, Beraldo AA, Villa TCS. Conhecimento de enfermeiros de unidades de atenção básica acerca da tuberculose. Cogitare Enferm. 2014;19(1):34-40. doi: 10.5380/ce.v19i1.35930

12. Andrade RPS, Maia VF, Queiroz RF, Carreiro GSP, Villa TCS, Pinto ESG. Professional contribution of primary health care for assisted self care to patients with tuberculosis. Revista de Pesquisa: Cuidado é Fundamental Online. 2016 ; 8(3):4857-4863. doi: 10.9789/2175-5361.2016. v8i3.4857-4863

13. Sobrinho ECR, Freitas KG, Figueiredo RM, Caliari JS. Tuberculosis in the family health strategy: the knowledge of community health agents. Rev Eletr Enf. 2013;15(2):41 6-421. doi: $10.5216 /$ ree.v15i2.16982
14. Sánchez AIM, Bertolozzi MR. Conhecimento sobre a tuberculose por estudantes universitários. Bol Pneum Sanit. $2004 ; 12(1): 17-24$.

15. Mussi TVF, Traldi MC, Talarico JNS. Knowledge as a factor in vulnerability to tuberculosis among nursing students and professionals. Rev Esc Enferm USP. $2012 ; 46(3): 696-703$. doi: $10.1590 /$ S0080-62342012000300023

16. Teixeira EG, Menzies D, Cunha AJLA, Luiz RR, RuffinoNetto A, Scartozzoni MS et al. Knowledge and practices of medical students to prevent tuberculosis transmission in Rio de Janeiro, Brazil. Rev Panam Salud Publica. 2008;24(4):265270.

17. Basu M, Das P. Assessment of knowledge regarding tuberculosis in the context of revised national tuberculosis control program among budding doctors. Chronicles of Young Scientists. 2014;5(1):59-64. doi: 10.4103/2229$\underline{5186.129340}$

18. Al-Jabri AA, Dorvlo ASS, Al-Rahb S, Al-Abri J, Al-Adawi S. Knowledge of tuberculosis among medical professionals and university students in Oman. East Mediterra Health J. 2006; 1 2(5):509-521.

19. Temesgen C, Demissie M. Knowledge and practice of tuberculosis infection control among health professionals in Northwest Ethiopia; 2011 . BMC Health Serv Res. 2014;1 4:593. doi: 10.1186/s12913-014-0593-2

20. Ministério da Saúde. Grupo Hospitalar Conceição. Tuberculose na Atenção Primária à Saúde. Porto Alegre: Hospital Nossa Senhora da Conceição; 2011.

21. Brasil. Ministério da Saúde. Portaria $n^{\circ} 1.602$, de 17 de julho de 2006. Institui em todo o território nacional, os calendários de Vacinação da Criança, do Adolescente, do Adulto e do Idoso.

22. Freitas IM, Crispim JA, Pinto IC, Villa TCS, Brunello MEF, Pinto PFPS et al. Knowledge And Perception About Tuberculosis Of Patients' Families Under Directly Observed Treatment At A Health Service In Ribeirão Preto-SP, Brazil, Brasil. Texto Contexto Enferm. 201 2;21 (3):642-649. doi: $10.1590 /$ S0104-07072012000300020

23. Vieira AA, Ribeiro SA. Noncompliance with tuberculosis treatment involving self administration of treatment or the directly observed therapy, short-course strategy in a tuberculosis control program in the city of Carapicuiba, Brazil. J Bras Pneumol. 2008;34(3):159-166. doi: 10.1590/S180637132008000300006

24. Montagna MT, Napoli C, Tafuri S, Agodi A, Auxilia $\mathrm{F}$, Casini $B$ et al. Knowledge about tuberculosis among undergraduate health care students in 15 Italian universities: a cross-sectional study. BMC Public Health. 2014;1 4:970. doi: 10.1186/1471-2458-14-970 